\title{
Proposal of character correction method using database
}

\author{
Takahiro Fujishima $^{\mathrm{a}}$, Ken Kariya ${ }^{\mathrm{a}}$, Lifeng Zhang ${ }^{\mathrm{a}, *}$ \\ ${ }^{a}$ Kyushu Institute of Technology, Kitakyushu, 800-0207, Japan \\ *Corresponding Author: zhang@elcs.kyutech.ac.jp
}

\begin{abstract}
Recently, labor shortages have occurred in various industries. In response to such labor shortages, improvement of various operations is required. In recent years, the Internet has developed rapidly, and information has been converted into digital data. That is, the information on the paper is converted into data and managed by the personal computer. That is because converting paper data into digital data enables spacesaving, quick search, and security measures. However, there are challenges in the character recognition rate when converting to digital data. There are various kinds of characters in the world, and it is very difficult to recognize them $100 \%$ with a perfect system. One of the essential tools for data conversion is OCR. Although OCR is a widely used optical character recognition system, it may be misrecognized depending on the environment and equipment. Although high-precision character recognition can be performed for characters which have good printing quality, the recognition rate will be low if low-quality characters such as fax and copied documents are used. If misrecognized, additional work will occur, and work efficiency will decline. This work targets on improving the character recognition rate for a paper printed order form sent by overseas FAX. In this research, we construct a misrecognition correction algorithm using a database.
\end{abstract}

\section{Introduction}

Converse paper printed characters to digital text data is still an important task that many companies are working on it. With the digitization of documents (paperless), companies can enjoy many benefits such as cost savings, operational efficiencies improvement, and risk management enhancement. Therefore, the accuracy of document digitization is an important topic ever before. There are many different types of documents in the world, and it is, in fact, impossible to recognize characters with $100 \%$ accuracy. Documents with print quality can be recognized characters with an accuracy close to $100 \%$, but low-quality characters such as fax and copy documents obtain a low recognition rate. By now, If the letters were misrecognized, people have to make artificial corrections. Therefore, this study proposes a new additional approach to correct the text database on the first stage OCR recognition result.

\section{Principle}

\subsection{Database}

A database ${ }^{(I I)}$ is a collection of information that is grouped into multiple pieces of data that meet a specific condition and then organized into a form that is easy to use later. The data is stored to be independent of the program using the data and can be accessed by multiple programs at the same time. A common and controlled approach is used to add new data, repair, and retrieve data present in the database. Structurally remember the data to be the basis for developing future applications. If the database is structurally distinct, one system will contain a collection of these databases. Therefore, if the printed document has fixed text items, the common knowledge base can be constructed by using a database. Then, using such common knowledge, the OCR result can be verified and corrected.

Also, a database that handles data based on a concept called a relational model, which is one of the database formats, is called a relational database. In a relational database, columns (columns) and records (rows) are assigned to data, and they are arranged in a table. Data can be easily extracted by rearranging the data around each column and record. Relational databases are currently the most widespread and often refer to relational databases in the case of databases. The system for operating and managing the relational database is called a relational database management system (RDBMS). Typical relational database management systems include Oracle Corporation Oracle, Microsoft Corporation SQL Server, MySQL, SQLite, PostgreSQL distributed as OSS, and others. Among them, SQLite is a database that gathers files and has a straightforward structure. 


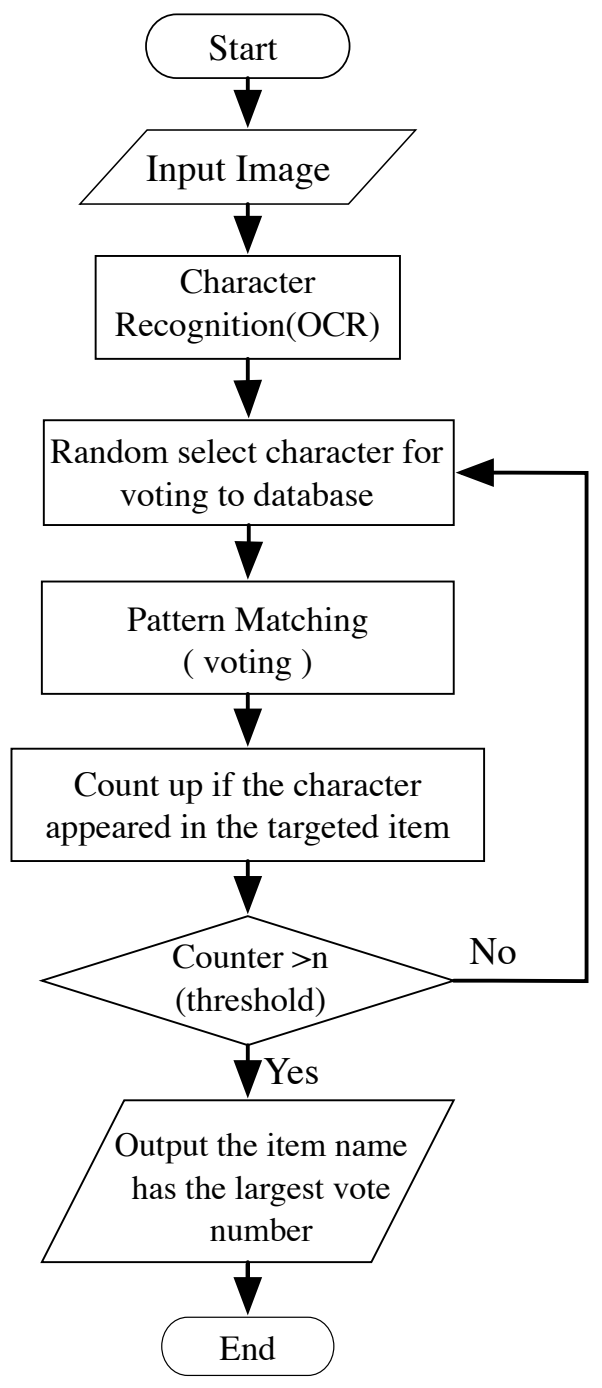

Fig. 1. Flowchart

Because databases are accessing differently from COBOL, C language, Java, Python, and other programming languages, an extension is needed using for the connection to the database in a programming language. Connect to the database using this extension module and communicate with the database using SQL. SQL is a database language for manipulating data created for relational databases, in response to instructions from users and systems, queries the relational database and returns the results. It does not compile but behaves like an interpreted language.

\subsection{OCR(Optical Character Recognition) $)^{(2)}$}

(a) Image capture

Because the target to recognize is a paper printed order form sent by overseas FAX, in this work a scanner or a camera is used to acquire the documents into the computer. (b) Layout analysis

Find the character part from the text region, the image region, and the ruled line of the document and decide the order to read. In this work, this part is done manually.

(c) Character segmentation

Focus on one text line and divide it into single characters. In this process, while moving the vertical lines from left to right, the number of intersections between the lines and characters is counted so that a histogram can be made, and it is determined the positions where have minimum local value is character boundary.

(d) Character recognition

Finally, check the font size, font type (Mincho, Gothic, Textbook, Textbook, etc.) and the collapsed characters. Following the normalization, feature extraction, matching, and knowledge processing, the characters were recognized.

\subsection{Pattern Matching with Database}

Pattern matching is a method of specifying whether or not a specific pattern will appear and where it appears when searching for data. For pattern matching of character strings, there are various character string searching algorithms such as $\mathrm{KMP}^{(\overline{3})}$ method and $\mathrm{BM}^{(\overline{4})}$ method in the search of fixed patterns. Many methods using regular expressions have also been proposed.

In this work, a voting approach is employed. The process is described below.

(1) Randomly select several characters(3 was used here) form the OCR recognition result, then voting if the item stored in the database include these selected characters.

(2) If the item includes all the selected characters, then count up the vote number of this item.

(3) Repeat the above procedure for enough times (100 times was used for this work), then get the statistical data, the most frequently voted item can be thought as the correct answer.

\section{Experiment}

Experiment is conducted according to the flowchart (Fig. (W) in the previous chapter. The input image uses the image shown in Fig. 2 .

For character recognition (OCR), learning data was created using fax print characters using jTessBoxEditor, and 


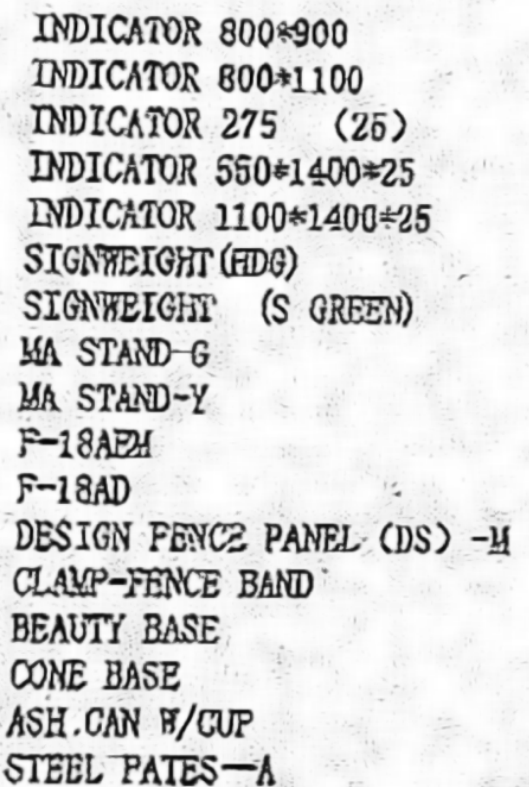

Fig. 2. Input image

Table 1.100 counts

\begin{tabular}{|c|c|c|c|}
\hline Extracted character/Correct search & 3 & 4 & 5 \\
\hline INDICATOR $800 * 900$ & 100 & 100 & 100 \\
\hline INDICATOR $800 * 1100$ & 100 & 100 & 100 \\
\hline INDICATOR $275(25)$ & 100 & 100 & 100 \\
\hline INDICATOR $550 * 1400 * 25$ & 100 & 100 & 100 \\
\hline INDICATOR $1100 * 1400 * 25$ & 100 & 100 & 100 \\
\hline SIGNWEIGHT (HDG) & 25 & 43 & 54 \\
\hline SIGNWEIGHT (S GREEN) & 100 & 100 & 100 \\
\hline MA STAND-G & 0 & 0 & 0 \\
\hline MA STAND-Y & 100 & 100 & 100 \\
\hline F-18AEM & 100 & 100 & 0 \\
\hline F-18AD & 100 & 100 & 100 \\
\hline DESIGN FENCE PANEL (DS) -M & 100 & 100 & 100 \\
\hline CLAMP-FENCE BAND & 100 & 100 & 100 \\
\hline BEAUTY BASE & 100 & 100 & 100 \\
\hline CONE BASE & 100 & 100 & 100 \\
\hline ASH CAN W/CUP & 100 & 100 & 100 \\
\hline STEEL PATES-A & 100 & 100 & 100 \\
\hline
\end{tabular}

OCR software (Tesseract-OCR) suitable for fax characters was used.

\section{Result and Consideration}

In existing OCR, almost all of the product names were recognized with mistaken characters, after applied the additional recognition method using the database voting, 15 items out
Table 2. 1000 counts

\begin{tabular}{|c|c|c|c|}
\hline Extracted character/Correct search & 3 & 4 & 5 \\
\hline INDICATOR $800 * 900$ & 100 & 100 & 100 \\
\hline INDICATOR $800 * 1100$ & 100 & 100 & 100 \\
\hline INDICATOR $275(25)$ & 100 & 100 & 100 \\
\hline INDICATOR $550 * 1400 * 25$ & 100 & 100 & 100 \\
\hline INDICATOR $1100 * 1400 * 25$ & 100 & 100 & 100 \\
\hline SIGNWEIGHT (HDG) & 3 & 17 & 66 \\
\hline SIGNWEIGHT (S GREEN) & 100 & 100 & 100 \\
\hline MA STAND-G & 0 & 0 & 0 \\
\hline MA STAND-Y & 100 & 100 & 100 \\
\hline F-18AEM & 100 & 100 & 0 \\
\hline F-18AD & 100 & 100 & 100 \\
\hline DESIGN FENCE PANEL (DS) -M & 100 & 100 & 100 \\
\hline CLAMP-FENCE BAND & 100 & 100 & 100 \\
\hline BEAUTY BASE & 100 & 100 & 100 \\
\hline CONE BASE & 100 & 100 & 100 \\
\hline ASH CAN W/CUP & 100 & 100 & 100 \\
\hline STEEL PATES-A & 100 & 100 & 100 \\
\hline
\end{tabular}

of 17 items could be recognized correctly. However, the erroneous search count of "MA STAND-G" was $100 \%$ in all cases of experiments 1 and 2 . This is probably due to failure to recognize "G" of "MA STAND-G". The experiment was carried out with setting the number of counts to 100 times, 1000 times, but the time takes to search all entered product names is about 1 second at the time of 100 counts and about 4 seconds at the time of 1000 times count. There was a difference of about 3 seconds. In addition, the number of erroneous searches was also larger when counting 1000 times than when counting 100 times, compared with the case of extracting characters in each case. Also, although it was output correctly, there was a character string with a small number of counts ( "F - 18 AEM"). This is also considered to be caused by lousy recognition of Tesseract-OCR.

\section{Conclusions}

In this research, we examined whether it would be possible to acquire the product name data of the correct character string by using SQLite as the database and performing pattern matching between the OCR product name and the product name data in the database. As a result, even if the product name includes misrecognized characters, it is possible to retrieve and output the correct product name data. 


\section{References}

(1) James Martin, Yoshihisa Kunitomo, and Misa Kubo: "Database [revised 2nd edition](in Japanese)", Japan Computer Society, 1983

(2) Character Recognition Technology(in Japanese), http: //mediadrive.jp/technology/techocr $07 . h t m I$, NTT data NJK co. Ltd., accessed: 2019.3.18.

(3) KMP Algorithm for Pattern Searching, https: //WWw.geekstorgeeks.org/kmp-algorithm-torpattern-searching/, A computer science portal for geeks, accessed: 2019.4.16.

(4) Boyer Moore Algorithm for Pattern Searching, https://www.geekstorgeeks.org/boyer-moorealgorithm-tor-pattern-searching/, A computer science portal for geeks, accessed: 2019.4.16.

(5) Comparing with pattern matching, https:// wWw.dbonline.jp/sqlite/select/index6.html, DBOnline, accessed: 2019.4.26. 